\title{
La Genèse dans la littérature martiniquaise contemporaine : souvenirs et devenir
}

\author{
Valentine Eugène*
}

\section{Gênesis na literatura Martinicana contemporânea: memórias e devir}

\section{Resumo}

O artigo examina o desdobramento das primeiras páginas da Bíblia na literatura Martinicana contemporânea. O gesto de apropriação e subversão do mito de Gênesis em La Vierge du Grand Retour (1996), de Raphaël Confiant, e na Biblique des latest gestes (2002), de Patrick Chamoiseau, cristaliza questões específicas das literaturas de língua francesa em um contexto pós-colonial: desenterrar o não dito de um processo histórico traumático; compor um mito original ausente; conceder às minorias um lugar sem precedentes na História e no Mito.

Palavras-chave: Antilhas, Gênesis, reescritura.

\section{Genesis in Contemporary Martinican Literature: Memories and Becoming}

\section{Abstract}

This paper proposes to examine the fate of the first pages of the Bible in contemporary Martinican literature. The gesture of appropriation and subversion of the genesis myth in La Vierge du Grand Retour (1996) by Raphaël Confiant and in Biblique des latest gestes (2002) by Patrick Chamoiseau crystallizes specific issues to French-speaking literatures in a postcolonial context: exhuming a traumatizing historical process; creating an original lacking-myth; giving the minorities a new place in the History and the Myth.

Keywords: Antilles, Genesis, rewriting.

* Doctorante contractuelle à l'université Sorbonne Université.

Email: eugene.valent@gmail.com 


\section{La Genèse dans la littérature martiniquaise contemporaine: souvenirs et devenir}

\section{Résumé}

Cet article propose d'examiner le devenir des premières pages de la Bible dans la littérature martiniquaise contemporaine. Le geste d'appropriation et de subversion du mythe génésiaque dans La Vierge du Grand Retour (1996) de Raphaël Confiant et dans Biblique des derniers gestes (2002) de Patrick Chamoiseau cristallise des enjeux spécifiques aux littératures francophones en contexte postcolonial : exhumer les non-dits d'un processus historique traumatisant ; composer un mythe originel manquant ; accorder aux minorités une place inédite dans l'Histoire et dans le Mythe.

Mots-clés: Antilles, Genèse, réécriture.

Si la Bible ${ }^{1}$ est aujourd'hui « un bien partagé des Antillais qui en usent à leur façon, développent leur foi et leur littérature selon leur génie propre » (GANNIER, 2016, p. 239), les relations du sous-ensemble continental américain avec ses pages furent loin d'être toujours aussi heureuses. Texte sacré, objet littéraire, modèle poétique, réservoir culturel, la Bible fut aussi, à un moment donné de l'Histoire, un instrument d'oppression et d'assujettissement. Les circonstances ayant conduit les Écritures jusqu'aux « confins du monde » révèlent à quel point quelques-unes des grandes histoires qu'elles racontent ont pu jouer un rôle capital dans les processus colonialistes et esclavagistes ${ }^{2}$. Transformé par la suite en "pièce maîtresse de la structure coloniale » (GANNIER, 2016, p. 238-239), que devient donc aujourd'hui le Livre dans les Antilles françaises ? Quel sens faut-il accorder, dans La Vierge du Grand Retour (1996) de Raphaël Confiant ${ }^{3}$ et dans Biblique des derniers gestes (2002) de Patrick Chamoiseau ${ }^{4}$, au geste d' "absorption » et de " transformation » ${ }^{5}$ de ce modèle si profondément ambivalent ?

1 Nous nous réfèrerons, tout au long de notre étude, à l'édition suivante : La Bible de Jérusalem (1998), L’École biblique de Jérusalem (trad. fr.), Paris, Pocket, coll. « Pocket », nº10456, Nouv. éd. rev. et cor., 2013.

2 Voir en particulier PrIOR M., Bible et colonialisme. Critique d'une instrumentalisation du texte sacré, Jourez P. (trad. fr.), Paris, l'Harmattan, 2003 et Hervieux G., L'Ivresse de Noé. Histoire d'une malédiction, Paris, Perrin, 2011.

3 Nous nous réfèrerons, tout au long de notre étude, à l'édition suivante : Confiant R., La Vierge du Grand Retour (1996), Paris, Gallimard, coll. «Folio », n4602, 2007. Nous utiliserons désormais le sigle suivant : VGR.

4 Nous nous réfèrerons, tout au long de notre étude, à l'édition suivante : CHAMOISEAU P., Biblique des derniers gestes, Paris, Gallimard, 2002. Nous utiliserons désormais le sigle suivant : BDG.

5 Nous reprenons ici la définition de l'intertextualité de Julia Kristeva : «tout texte se construit comme mosaïque de citations, tout texte est absorption et transformation d'un autre texte » (Kristeva J., Séméiotikè. Recherches pour une sémanalyse (1969), Paris, Éditions du Seuil, coll. «Points », nº9, 1978, p. 85). 
La Vierge du Grand Retour démarque nettement l'hypotexte biblique. Si le titre en lui-même ne renvoie pas directement aux Écritures - l'évènement auquel celui-ci fait allusion n'est pas contenu dans la Bible, mais dans l'Histoire -, la structure interne du roman, en revanche, s'affiche comme son parfait décalque. Se succèdent ainsi «La Genèse », «L'Exode », "Le Lévitique », «Les Nombres », « Le Deutéronome », «L'Ecclésiaste », et bien d'autres encore, jusqu'à «L'Apocalypse » finale. La dénomination des chapitres constitue ainsi un « indice officiel »(GENETTE,1982,p. 436) précisant systématiquement la relation du contenu de la narration à l'intertexte biblique. Raphaël Confiant, pourtant, ne choisit pas de situer l'action de ses personnages dans la légendaire Canaan ou dans la Galilée mythique. L'écriture concentre son attention sur une manifestation religieuse réelle qui fit date dans les mémoires martiniquaises : le pèlerinage de Notre Dame de Boulogne à travers l'île de la Martinique (1948) ${ }^{6}$. Le choix thématique est loin d'être anecdotique. Celui-ci permet de rendre compte, dans une veine « ludique et picaresque » (FRATTA et GiAUfret, 2009, p. 5), des problématiques traversées par l'île à cette époque, et de questionner le rapport établi entre le DOM et sa Métropole deux ans seulement après la date de la départementalisation (1946). Les interrogations formulées au départ, plutôt neutres, prennent rapidement une tournure polémique.

Contrairement à Raphaël Confiant, Patrick Chamoiseau privilégie un titre qui, clairement, exhibe la référence aux Écritures : Biblique des derniers gestes. Pourtant, l'usage que l'écrivain martiniquais réserve au vocable interroge. Si, dans la phrase clausule du roman, les conditions d'émergence du lexème favorisent l'interprétation adjectivale ${ }^{7}$, dans le titre en revanche tout donne à croire que Biblique puisse être entendu comme un substantif. Favorisant le rapprochement de cette œuvre et du « Grand Code de l'Art» ${ }^{8}$, le glissement grammatical participerait ainsi à la création d'une « nouvelle catégorie de récit »(CHAuvin, 2008, p. 126) inspirée de la poétique biblique. Le roman

\footnotetext{
6 Sur le pèlerinage accompli en 1948 à la Martinique, voir Pérouas L., « Le grand retour de Notre-Dame de Boulogne à travers la France (1943-1948). Essai de reconstitution », Annales de Bretagne et des pays de l'Ouest, Tome 90, numéro 2, 1983.

«M. Balthazar Bodule-Jules était devenu bien plus qu'un simple rebelle, sans doute un grand guerrier, et [...] ses déplacements empreints de majesté gravaient dans nos mémoires, comme pour l'ouvrir et l'achever, la démesure biblique de ses derniers gestes. » (BDG, 773).

$8 \quad$ L'expression est de William Blake. Cette dernière s'est vue reprise en 1982 par Northrop Frye pour intituler l'un de ses ouvrages, Le Grand code [The Great code], trad. de l'anglais Catherine Malamoud, Paris, Seuil, coll. « Poétique », 1984.
} 
dans lequel Patrick Chamoiseau règle moins la mesure de son écriture sur les

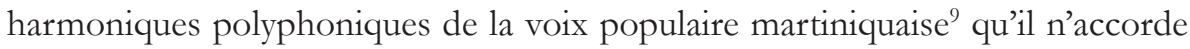
son Verbe aux consonances symphoniques du monde se verrait donc placé sur un plan concurrentiel à l'ouvrage fondateur de la conscience occidentale. Dans cette épopée en clair-obscur, qui consigne les exploits de Balthazar Bodule-Jules et narre son agonie, la recherche - ou l'élucubration - d'une mémoire fondatrice crée l'originalité d'un récit qui, plus que les événements, enchaîne les épiphanies. De guerre en guerre, de révolution en révolution, les épreuves du « guerrier» de «toutes époques » et de « tous lieux » $(B D G, 27)$ se comprennent et s'écrivent autrement jusqu'à ce qu'émerge la conscience d'un insu partagé.

L'ambition totalisante de la plume chamoisienne, en rassemblant et en condensant un ensemble d'« histoire-histoires » (BDG, 692), élargit bientôt les problématiques martiniquaises aux perspectives du monde, et à ses origines. Tout comme La Vierge du Grand Retour, Biblique des derniers gestes, en plusieurs endroits stratégiques de sa narration, actualise des souvenirsvivaces de la Genèse. C'est pourquoi nous concentrerons notre attentionle devenir des premières pages de la Bible et du grand mythe de création qu'elles élaborent. Le travail que nous engageons ne vise pas seulement à décrire l'imaginaire pour lui-même. En privilégiant une approche d'inspiration mythocritique ${ }^{10}$ qui gardera trace du séisme épistémologique que représentent les démarches postcoloniales ${ }^{11}$, nous appréhenderons les divers échos de Gn1 rencontrés

9 C'était notamment le cas dans les premiers romans urbains de Patrick Chamoiseau. Voir en particulier Chamoiseau P., Texaco, Paris, Gallimard, 1992 et Chamoiseau P., Chronique des sept misères, Paris, Gallimard, 1986.

10 Voir la façon dont Danièle Chauvin, dans «Bible et Questions de mythocritique », retrace l'émergence de cette approche et définit cette discipline. Chauvin D., «Bible et Questions de mythocritique ", Questions de mythocritique. Dictionnaire, Chauvin D., Siganos A. et Walter PH. (dir.), Éditions Imago, 2005, p. 47.

11 En 1999, dans un ouvrage qui avait pour vocation d'interroger les spécificités des littératures francophones et de questionner ses rapports au postcolonialisme, Jean-Marc Moura rappelait le sens historique généralement accordé au vocable post-colonial: " 'Postcolonial' désigne [...] le fait d'être postérieur à la période coloniale » (Moura J.-M., Littératures francophones et théorie postcoloniale (1999), Paris, Presses universitaires de France, coll. «Quadrige Manuel », 3e éd., 2013, p. 10). Son presque parfait homonyme postcolonial engloberait quant à lui tout «texte écrit par des auteurs venant de pays marqués par l'histoire coloniale, principalement les textes concernés par les actions et le legs du colonialisme, dans le passé comme actuellement » (McLeod J., Beginning Postcolonialism, Manchester, Manchester university press, coll. «Beginnings », 2000, p. 33.). À ce titre, le postcolonialisme devrait idéalement être compris comme une situation d'écriture. 
dans nos textes comme l'indice d'un projet sémantique beaucoup plus vaste, engageant l'inscription de l'Histoire - et de ses sujets parfois oubliés - dans le mythe et du mythe dans l'Histoire.

\section{Au Commencement était le crime}

En apportant à l'homme des réponses sur la question de l'origine, les mythes cosmogoniques, au rang desquels il convient de ranger Gn1, « déborde[nt] les limites de l'expérience pour rejoindre ce qui, précédant celle-ci, la fonde et lui confère un sens »(Pelletier, 1995, p. 28). À ce titre, ils se présentent moins comme "un langage des faits » que comme "un langage qui élabore des significations» (PELLETIER, 1995, p. 28). Les récits de création, eux, fondent avant tout l'historicité d'une communauté, et légitiment l'existence des groupes sociaux. Un lien extrêmement ténu rattache donc les mythes cosmogoniques et les récits de création à la mémoire. En «port[a]nt [...] témoignage de l'identité» (CHAuvin, 2005, p. 230), tous deux jouent un rôle prééminent dans la question - complexe, mais essentielle en contexte postcolonial $^{12}$ - de l'autodétermination des peuples. L'un et l'autre discours devraient idéalement se concevoir «comme trace de faits » et «comme mouvement». L'histoire qu'ils racontent ne relève pas d'une mémoire « close » et «définitivement arrêtée»(CHAUvin, 2005, p. 230), mais participe de la dynamique même de l'Homme et de sa quête de sens. Ce faisant, la mémoire, tout à la fois tournée vers ce qui est advenu et vers ce qui doit advenir, révèle la nature profondément dialectique de son fonctionnement. Le passé n'est pas lettres mortes : il est envisagé comme un socle de départ et un comme tremplin pour l'avenir sur lequel s'appuie une mémoire vive, en perpétuel devenir. À cet égard, une question mérite d'être posée : qu'advient-il lorsque ce passé est traumatisant?

12 Les Petites Antilles, colonisées par la France en 1635, restèrent tardivement des domaines coloniaux. Il faudra attendre 1946 pour que leur statut post-colonial soit enfin négocié. La départementalisation, qui se présentait aux yeux de Victor Schœlcher comme le point d'aboutissement logique de l'histoire institutionnelle et la meilleure façon de garantir les droits des affranchis, ne consacra donc pas l'autonomie de l'archipel. La mise en place d'une politique d'assimilation ne fit d'ailleurs que renforcer les liens culturels rattachant les nouveaux DOM à leur Métropole. Aux yeux de certains auteurs, de tels liens continueraient d'enchaîner insidieusement les Antillais à leur ancien statut de colonisés. Patrick Chamoiseau affirmait ainsi au début des années 1990 que les départements d’outre-mer représentent « les dernières colonies françaises» (Chamoiseau P., dans Confiant R., Aimé Césaire. Une traversée paradoxale du siècle, Paris, Stock, coll. «Échanges », 1993, 4e de couverture). 
Les processus historiques des Antilles témoignent d'une tragique réalité : lorsqu'au fondement d'une communauté se rencontre un crime, tout discours de l'origine semble difficile, sinon impossible, à formuler. Très tôt après sa découverte par les Occidentaux, la Martinique vit sa population locale décimée. Si les premiers rapports entre les colons français et les natifs de l'île furent pacifiques, divers conflits devaient surgir par la suite. Les relations entre les deux peuples, largement dégradées depuis les années 1640, s'envenimèrent définitivement à la mort du gouverneur du Parquet. Une guerre éclata. La plupart des esclaves des Caraïbes furent exterminés. L'économie sucrière, qui nécessitait une main-d'œuvre abondante, connut alors sa première crise. On chercha de nouveaux captifs. Les expéditions outre-Atlantique se multiplièrent ${ }^{13}$. Les conséquences tragiques du commerce humain engagé sur plusieurs siècles entre les côtes africaines et les rives caribéennes ne sont plus à démontrer : exploitation et dévastation de l'Homme, de sa liberté, de sa dignité - et de son identité. L'« Arrachement au pays natal » et " l'expérience du milieu» (Clavaron, 2012, p. 30) rompit brutalement tous les liens avec l'Afrique. Aucun mythe fondateur créole n'est jamais - ou n'a jamais pu - venir rétablir ou compenser pareille déchirure. Pour Raphaël Confiant, «s'il existe bien deux termes parfaitement antinomiques dans l'univers antillais, ce sont bien ceux de 'mythe' et de 'créole'» (Confiant, 1998, p. 9). Comme l'analyse Édouard Glissant, «aux origines de l'Antillais ou du Caribéen il y a non une Genèse, mais un fait historique combien de fois établi, et combien de fois raturé de la mémoire publique, qui est la traite négrière »(GLISSANT, 1998, p. 265).

Cette violence historique, Patrick Chamoiseau en rend particulièrement compte dans le récit qui retrace la deuxième naissance de son protagoniste ${ }^{14}$,

13 L'année 1755 compte une cinquantaine de traversées ; l'année 1783 en comptera, elle, plus d'une centaine. Voir Butel P., Histoire des Antilles françaises XVII $\mathrm{e}^{\mathrm{e}} X \mathrm{X}^{\mathrm{e}}$ siècles (2002), Paris, Perrin, coll. «Tempus », n¹74, 2007, p. 212.

14 Il n'y a pas, dans Biblique des derniers gestes, un seul récit donné pour Genèse, mais trois récits (BDG, 52-55, 55-66, et 70-80). Le procédé d'interpolation auquel recourt l'écrivain martiniquais s'apparente à celui mis en œuvre par les auteurs de la Bible hébraïque qui, eux aussi, conjuguèrent ensemble des récits parallèles de la Création du monde. Dans notre roman, les phénomènes d'interpolation sont d'ailleurs courants. Comme dans les Écritures, la cohérence de l'ensemble repose alors moins sur le respect d'une logique linéaire que sur la volonté d'englober une connaissance plus large et plus complète d'une vérité. Sur la façon dont «l'art du récit composite » aurait permis d'assurer l'ampleur de la vision biblique, voir Alter R., L'Art du récit biblique (1981), Lebeau P. et Sonnet J.-P. (trad. fr.), Bruxelles Namur, Lessius Presses universitaires, coll. « Le livre et le rouleau », nº4, 1999, p. 179-209. 
Balthazar Bodule-Jules (BDG, 56-66). Le cadre spatio-temporel dans lequel se déroule l'action - le fond d'une cale d'un bateau négrier - et la douleur ressentie par le personnage à la simple évocation des faits transforment l'épisode génésiaque en un véritable "songe d'apocalypse » $(B D G, 62)$ dont l'horreur atteindra son paroxysme dans l'image hyperbolique de ces « deux cents rebus de chairs, $[\ldots]$ flott[ant] dans l'éclat métallique des icebergs de peau noire et de caillots de sel » $(B D G, 62)$. L'abondance des détails numéraux et la saturation adjectivale, qui contribuent à caractériser l'« enfer sans nom» $(B D G, 60)$, composent un tableau saisissant situé à mi-chemin entre le cauchemar éveillé et la réalité qui se dérobe. Le texte, saturé par les isotopies de la violence et de la souillure ${ }^{15}$, emprunte au paradigme biblique certains de ses mythèmes et la forme de ses discours ${ }^{16}$. Ceux-ci, pourtant, sont moins à rechercher dans les premières lignes du Livre que dans ses derniers chapitres.

Comme n'importe quelle phraséologie apocalyptique, celle de l'écrivain martiniquais intègre tous les types de morts : les morts lentes, sujettes à la décrépitude ${ }^{17}$; les morts violentes, à l'image du capitaine faisant jeter pardessus bord « une rangée de captifs enferrés l'un à l'autre » $(B D G, 61)$; les morts «totales» (Chauvin, 1994, p. 107) ; les morts « limitées» (Chauvin, 1994, p. 107), comme celle qui, nous dit-on, cherchait insidieusement à aspirer Balthazar Bodule-Jules et persistait à demeurer collée « en ventouse » sur sa peau $(B D G, 59)$. La notion d'oubli, centre névralgique du récit ${ }^{18}$, signe la concurrence de deux types de destructions : l'une est physique, effective, concrète ; l'autre est symbolique. La perte d'une mémoire et d'une identité entérine, avant la maladie ou la maltraitance décrite par le texte, la lente mais irrépressible désagrégation du héros. Son « corps », nous signale-t-on, eut conscience de lui-même comme « d'un chyme de chair et d'os, de langues tombées, de valeurs empêchées, de dieux pâlis, de traditions en effiloches qui

15 On relève, entre autres occurrences, les substantifs «vomissures » (BDG, 60) et « excréments » $(B D G, 60)$. Ces dernières concourent à ramener l'humanité à ce qu'elle a de plus bas, de plus trivial, et de plus animal.

16 Se trouve ainsi actualisé, dans un contexte proprement dantesque, au moment d'évoquer « la maladie de toutes les maladies » $(B D G, 59)$, un hébraïsme de haute solennité.

17 De telles morts sont suggérées par le motif de la maladie.

18 Dans l'épisode narratif considéré, le thème de l'oubli demeure central : «Ses frères [...] l'avaient forcé à tourner sept fois autour du grand arbre de l'oubli » $(B D G, 59)$; «cette terre africaine avait achevé de s'épuiser en lui à mesure que le navire, quittant la barrière de corail, avait déployé en direction des terres nouvelles le désir de ses voiles » $(B D G, 59)$; «Son corps, captif dans cette cale, se souvenait à peine de sa terre africaine » $(B D G, 59)$. 
peuplaient ses cellules tétanisées » $(B D G, 59)$. Le motif du vide ${ }^{19}$, chromatisé par le noir - un noir désigné comme «stomacal » $(B D G, 59)$ et «sans passé » (BDG, 59) - rend compte de « l'expérience du gouffre » (GLissant, 1990, p. 18$)^{20}$. La dépossession de sa langue, de ses valeurs, de ses croyances et de ses traditions, toutes englouties dans le ventre sans fond d'un navire anthropophage, signe ici la mort du sujet. L'infernale traversée réduit toute humanité à des « rebuts de chairs » (BDG, 63).

La connotation eschatologique du texte n'est ni anodine, ni anecdotique : elle traduit la nécessité et l'urgence qu'il y aurait, face à l'épreuve de l'« attentat colonial» (Chamoiseau, 1997, p. 200), donnée pour si terrible qu'elle dépasse les lois transcendantales du langage et reste de l'ordre de l'ineffable en se présentant comme une « horreur aphone » (BDG, 57), de partir en quête d'un nouveau référent symbolique situé dans cette expérience historique même et non plus, comme avait jusque-là tâché de le faire les poètes de la Négritude, dans un ailleurs géographique et temporel : l'Afrique-mère. La plupart des discours articulés par les auteurs de la Créolité ou de l'Antillanité autour de la question de l'origine ${ }^{21}$ se réduisent d'ailleurs à une réitération de structures négatives. Celui de Patrick Chamoiseau (1997, p. 203) n’échappe pas à la règle :

Là, aucune de ces Genèses traditionnelles qui fondent les ethnies, les territoires, les identités anciennes, la belle Histoire commune. Pas de discours d'origines. Pas de mythe fondateur général. Pas de sacralisation d'un commencement quelconque.

Pour Noémie Auzas (2011, p. 171), la conclusion du phénomène syntaxique est évidente : le procédé place l'aire créole « sous le signe d'un

19 Pour Liviu Lutas, le motif du « vide » reste étroitement associé à la modalité narrative du fantastique et à l'écriture de l'Histoire. Voir Lutas L., Biblique des derniers gestes de Patrick Chamoiseau, Fantastique et Histoire, Lund, Suède, Media-Tryck, coll. «Études romanes de Lund », n82, 2008.

20 L'expression est d'Édouard Glissant. Celui-ci assimile d'ailleurs lui aussi, dans « la barque ouverte », la cale négrière à un «ventre » : «Le ventre de cette barque-ci te dissout, te précipite dans un non monde où tu cries » (Glissant E., Poétique de la Relation, Paris, Gallimard, 1990, p. 18-19).

21 L'Antillanité est formalisée par Edouard Glissant (voir Le Discours antillais), et la Créolité par Jean Bernabé, Patrick Chamoiseau, Raphael Confiant (voir Éloge de la créolitê). Ces deux tendances contestent la vision d'un « monde noir » de la Négritude, jugée trop monolithique. Intégrant l'apport multiculturaliste et multilinguistique des Caraïbes, elles ne prônent plus la redéfinition de l'homme noir à partir de ses racines. 
manque ». Ce dernier rend difficile, sinon impossible, l'existence d'une collectivité.

Dès lors, l'enjeu de l'écrivain serait de parvenir à créer, en réinvestissant l'imaginaire des origines bibliques, ce fameux mythe fondateur manquant, et, grâce à lui, à former une «nouvelle entité collective »(CHAMOISEAU, 1997, p. 202). L'élaboration d'un tel mythe permettrait ainsi de relier $^{22}$ les membres de la communauté désunie. L'auteur, envisagé par Chamoiseau comme un « inventeur de peuple» (Chamoiseau, 1997, p. 170) doit donc, avant toute chose, devenir un "inventeur de mythe ». La multiplication des emprunts bibliques témoignerait alors des efforts des créolistes pour (re)fonder une origine, (re)construire une généalogie, et (re)conquérir une légitimité. En dépit de son ancrage apocalyptique, le récit de la deuxième naissance de Balthazar Bodule-Jules conserve les spécificités d'une véritable genèse, et s'acquitterait à ce titre des mêmes fonctions que n’importe quel récit de création.

\section{(Re)construire le mythe originel}

La «Genèse " ${ }^{23}$ de Patrick Chamoiseau s’interprète comme la relecture mythique d'un transport subi dans l'Histoire. Un tel trajet, incontestablement négatif au départ, tend toutefois, au fur et à mesure de sa narration, à changer de valeur. Le renversement axiologique opéré par l'auteur prend appui sur la valorisation de l'un des quatre éléments essentiels de celui-ci. Ce qui, au cours du récit, finira par faire l'objet d'une réévaluation positive n'est ni le point d'origine - même s'il nous est initialement dit que «Cette évocation de la Traite des nègres comme douloureuse Genèse permettait à $M$. Balthazar Bodule-Jules [...] de se relier de manière indéfectible à l'Afrique », son Paradis perdu $(B D G, 58)$; ni le point d'arrivée - puisqu'au terme du voyage une vie de servitude attend le personnage ; ni même la trajectoire qui, en tant que ligne décrite d'un ancrage à un autre, permettrait de mesurer tragiquement l'écart entre l'ancienne condition d'homme libre et la nouvelle situation

22 «Je n'ai pas de solution collective à l'oppression esclavagiste car le collectif n'existe pas encore, il me faut d'abord relier ces hommes émiettés » (CHAMOISEAu P., Écrire en pays dominé, op.cit., p. 170).

23 Le terme même de "Genèse ", d'ailleurs, est convoqué dans le texte à plusieurs reprises. Ainsi, « M. Balthazar Bodule-Jules se revendiquait aussi, avec la même emphase, d'une Genèse autre, et pour le moins inattendue » $(B D G, 56)$. On soulignera l'importance de la majuscule, laquelle nous invite à dépasser l'acception usuelle du terme en nous renvoyant au livre de la Création biblique. 
d'esclave - mais le mouvement en lui-même et pour lui-même, dépris de toute orientation et de toute finalité.

Chamoiseau nous invite ainsi à relire le moment de la Traversée, cette phase obscure et tragique de l'histoire des Antilles et d'une grande partie des Amériques, non plus comme un trajet - ou une Chute originelle -, mais comme un mouvement pur qui, détaché de ses motivations premières, et arraché des conditions de sa réalisation, constitue, en tant qu'entre-deux, le point cristallisateur d'une «bascule vers des possibles inouïs » (CHAMOISEAU, 1997 , p. 203). C'est finalement cette bascule, autrement dit la dynamique transférentielle ${ }^{24}$ qui, dans le texte, fait l'objet d'une réelle mythification et permet d'effectuer une transmutation des valeurs propres à l'expérience traumatique. Dès lors, le récit de la deuxième naissance de Balthazar BoduleJules répondrait bien de l'ambiguïté des récits eschatologiques, lesquels « font état d'un anéantissement » certes, mais avec « la présupposition d'une nouvelle création »(GIRAUD, 2005, p. 367).

Le texte a pour spécificité de ne pas inscrire le principe de (re) création et/ou de (re)naissance dans une linéarité temporelle, mais sur un axe équinoxial sur lequel pourrait se (re)mesurer la valeur d'une relation inédite - aux éléments et aux évènements - devant être avant tout pensée comme interrelation et corrélation :

[Balthazar Bodule-Jules] avait entendu, tout autour et en lui-même, mêlé aux désenchantements de sorcière du navire, l'immémorial fracas de la vie et de la mort qui, une fois encore et à jamais, s'affairent au fourneau des créations nouvelles. M. Balthazar Bodule-Jules se déclarait né là, au pile-exact de cette conscience. (BDG, 60)

La « vie » et la «mort» ne s'opposent ni ne se succèdent. Concomitantes - la conjonction « et », qui a davantage la valeur d'une adjonction que celle d'une succession immédiate, assure d'ailleurs la liaison des deux termes et souligne syntaxiquement le principe de simultanéité-, elles deviennent sujet unique du verbe actif «s'affairer ». Le récit de cette singulière Genèse nous confronte à l'expérience d'une création continue au sein de laquelle le principe de dilution est immédiatement contrebalancé par celui d'une expansion.

24 La Traite esclavagiste est d'ailleurs appréhendée par Édouard Glissant comme une « déterritorialisation des hommes et des langues vers l’Ouest ». Voir Glissant E., Poétique de la Relation, op.cit., p. 16. 
Ce n'est pas tant la mort - physique ou symbolique - du « vieux guerrier » qui rend possible cette (re)création ou cette (re)naissance, mais la prise de conscience de l'indissociabilité de ces deux schèmes contradictoires. Le moment véritablement génésiaque de l'œuvre coïncide d'ailleurs avec l'expérience éprouvante mais inouïe de l'endurance des contraires ${ }^{25}$, comme si le personnage reconnaissait en eux, à cet instant précis, un moteur - voire le seul moteur - apte à générer de l'harmonie là où, en apparence, il n'y aurait que chaos. Une telle expérience arrache en lui le sentiment de l'incohérence de la condition humaine. La lutte, mais aussi la rencontre et le mouvement, sont ainsi placés, dans la cosmogonie chamoisienne, au cœur de l'univers. De sorte que naître au monde, dans cette Genèse apocalyptique - ou cette Apocalypse génésiaque - équivaut à concevoir - ou vivre, ou éprouver - le monde comme Relation ${ }^{26}$, autrement dit comme une nécessité composée en perpétuelle recomposition.

La prise de conscience du protagoniste correspond donc à un moment de «pile-exacte » qui, en lui permettant d'" ouvrir les yeux et les oreilles » $(B D G, 62)$, rend possible sa « redécouverte du monde » (BDG, 62). C'est seulement à partir de cette conscience aiguë de la simultanéité des contraires, conscience fragile et fugace, proche d'une expérience - d'ordre quasi mystique ${ }^{27}$ - de l'absolu, que pourront être octroyées à l'agonisant des « oreilles neuves » $(B D G, 62)$ et une nouvelle qualité de présence au monde. La (re)naissance de Balthazar Bodule-Jules coïncide avec un temps

25 Chamoiseau est un grand lecteur d'Héraclite. Dans L'Empreinte à Crusoé, l'écrivain, qui revisitera le mythe littéraire moderne de Robinson, multipliera les références au penseur. Voir Chamoiseau P., L’Empreinte à Crusoé, Paris, Gallimard, 2012.

26 La notion de Relation est un point focal de l'œuvre du poète, écrivain et philosophe martiniquais (voir notamment sa Poétique de la Relation. Poétique III, Paris, Gallimard, 1990). Dans une interview accordée à Anne Adler, Édouard Glissant englobait sous ce terme l'idée d'une «quantité finie de toutes les particularités du monde ». Cette dernière résulte d'une modalité idéale de la rencontre, définie par l'auteur comme « notre forme d'universel aujourd'hui », comme " notre manière [...] d'aller vers l'autre et d'essayer de se changer en échangeant avec l'autre sans se perdre ni se dénaturer » (GLISSANT E., interview réalisée par AdLer A. dans «L'invitation au voyage », 22 novembre 2004).

27 L'expérience sensorielle du toucher produit la vision, au moment où la " chair glaciale », objet extérieur, entre en contact avec le corps du personnage et frappe ses sens : «Le contact avec la chair glaciale du mort l'avait éclaboussé d'une lucidité barbare, solaire et nocturne, en devenir imperturbable. » $(B D G, 60)$. Le contact génère alors une autre vision, au cours de laquelle une réunion des contraires est opérée (le texte évoque une «lucidité [...] solaire et nocturne »). Ce faisant, le personnage fait l'expérience subjective d'une réalité objective - qui n'est pas, bien sûr, celle de Dieu, mais celle d'une autre forme d'absolu. 
d'« illumination $»^{28}$. À ce titre, elle se situe elle-même dans un espace-temps nécessairement transitoire et conjoncturel. Ce fameux temps d'« illumination », seul élément, peut-être, durablement valorisé par la narration, laisse alors entrevoir les contours d'un «paysage-chaos» figurant l'ouvert du « ToutMonde $»^{29}$ dont la complexité avait été intuitivement saisit dès l'antiquité par Héraclite.

Si les jeux d'échos et de correspondances entre les premiers chapitres de la Bible et la Création composée par Patrick Chamoiseau restent manifestes, plus grande encore se révèle l'affinité du récit élaboré par Raphaël Confiant dans La Vierge du Grand Retour avec les Écritures. Le roman s'ouvre sur une pure parodie de Gn1. L'auteur ne se contente pas d'emprunter au modèle sa lettre seule : il reprend aussi à son compte sa structure narrative, articulée par la reprise des trois refrains « Dieu dit», «Dieu appela », et « il en fut ainsi ». Dans l'hypotexte biblique, les deux premiers versets ${ }^{30}$ remplissent une fonction de préambule, et renvoient furtivement au mystère d'avant même le commencement. Le «vide », les «ténèbres » et l'« abîme » conduisent notre imagination jusqu'aux limites du connaissable. L'écrivain martiniquais inaugure sa propre « Genèse » (VGR, 15-16) par un curieux liminaire :

Au commencement, Yabvé Dieu créa le Morne Pichevin et la Cour Fruit-à-Pain au beau mitan de Fort-de-France ${ }^{31}$. (VGR, 15)

28 La deuxième naissance de Balthazar Bodule-Jules revêt, à bien des égards, l'apparence d'une révélation religieuse. On notera l'importance du thème de la lumière. D'une manière générale, c'est l'ensemble de cette naissance au fond de la cale d'un bateau négrier qui prend la forme d'une vision. L'esprit de Balthazar Bodule-Jules ne se représente pas uniquement sous une forme sensible, concrète, une situation dont le personnage n'a pourtant pas eu l'expérience directe. La force imaginative de l'agonisant, dans une véritable "vision prophétique du passé ", nous est présentée comme faculté d'évoquer, sous forme d'images mentales fortes, d'un réalisme saisissant et instinctif, l'insu partagé des descendants d'esclaves : l'imagination, présentée comme visionnaire, devient ici mémoire transgénérationnelle et cellulaire.

29 Nous empruntons ici à Edouard Glissant le titre de son roman Tout-Monde (1995), et celui de son essai Traité du Tout-Monde. (Poétique IV) (1997).

30 «Au commencement, Dieu créa le ciel et la terre. Or la terre était vide et vague, les ténèbres couvraient l'abîme et un vent de Dieu agitait la surface des eaux. » (Gn1, 1-2).

31 Nous conservons la typographie originale. Chaque chapitre de La Vierge du Grand Retour est en effet inauguré par un segment en italique qui, tout en révélant son intimité avec les Écritures, en marque les écarts : le récitant martiniquais, au moyen de modifications minimes, détourne alors significativement la lettre première vers un autre objet, et lui impose une tout autre signification. 
La formule est proche du modèle, mais pas identique à lui : il faut enregistrer la présence d'un hiatus entre le discours biblique et celui de Confiant qui, au lieu d'ouvrir « une fenêtre sur l'énigme du commencement antérieur au commencement même de la création » (PELLETIER, 1995, p. 31), nous ramène promptement dans un espace géographiquement identifiable. Aux lexèmes immémoriaux sont substitués des toponymes réels. Le «Morne Pichevin » se subroge au « ciel », la « Ravine Bonillé» aux « eaux », la "Cour Fruit-à-Pain » à la "terre ». L'ensemble des éléments qui, dans le premier chapitre de la Genèse, référaient au gros-œuvre cosmique, se trouvent, sous la plume de Raphaël Confiant, interchangés avec des quartiers de Fort-deFrance. Les réalisations effectives du Verbe divin sont ancrées dans un cadre énonciatif bien spécifique : celui de la Martinique.

Contrairement au paradigme biblique, la Genèse de La Vierge $d u$ Grand Retour ne met pas au premier plan la thématique temporelle, mais celle de l'espace. Dans le texte de référence, la première intervention divine, mentionnée dès le troisième verset, consiste à faire surgir la lumière. À l'appréciation de Dieu, qui « vit que la lumière était bonne »(Gn I, 4), s'ensuit un acte de séparation (Gn I, 4). La distinction de la lumière et des ténèbres nous suggère que la question du temps, comme celle de son instauration, relève d'une interrogation majeure dans l'hymne étiologique. Chez Raphaël Confiant en revanche, la première intervention divine concerne le surgissement de la «fraîcheur» :

Or une chaleur sans pareille régnait sur la terre, des échardes de feu tournoyaient au-dessus des eaux glauques de la Ravine Bouillé.

Yabvé Dien dit : "Que la fraîcheur soit! 》 et la fraîcheur fut. Le boulevard de La Levée et la place de La Savane s'ornementèrent de tamariniers géants. Des halliers poussèrent à la venvole au flanc de tous les quartiers qui saturaient la ville. (VGR, 15)

Le référent perturbe fortement la signification première des versets génésiaques. Priment, dans cette singulière Genèse, une problématique nouvelle, essentiellement liée aux conditions bioclimatiques des Antilles, et la « thématique de la cartographie ». Selon les théoriciens du Vieux Commonwealth, cette dernière intègre la liste des « sujets fertiles » de la littérature postcoloniale (BARDolph, 2002, p. 28). La Genèse de Raphaël Confiant, qui trace de nouvelles frontières de la Création biblique et nomme, à sa suite et selon ses propres modalités, certains lieux spécifiques, se fait 
«prise de pouvoir »(BARdolph, 2002, p. 28). La stratégie subversive que l'écrivain déploie dans ces lignes s'interprèterait dès lors comme la(ré) appropriation d'un lieu dont il se sent un « (éternel ?) locataire » (CONFIANT, 2005 , p. 180) et, par là-même, comme pied de nez fait à l'histoire coloniale. Le détournement des premières pages du Livre permettrait simultanément d'élaborer un mythe créole de l'origine qui, contrairement au récit fondateur de Patrick Chamoiseau, occulterait volontairement le traumatisme de la Traversée.Une telle recontextualisation de la Genèse permet de composer une contre-Bible au sein de laquelle peuvent enfin se lire et se partager les origines sacrées - ou pseudo-sacrées - d'une geste créole.

Pour autant, la présence d'un certain nombre d'éléments nous invite à penser que l'écrivain martiniquais ne tourne pas uniquement son regard vers une tradition pour rencontrer l'inspiration : les transformations qu'il impose au substrat biblique manifestent, certes, la volonté d'établir les fondements imaginaires et symboliques d'une nouvelle présence au monde du sujet martiniquais, mais aussi, et peut-être surtout, celle de jouer avec son système de représentations en vue de réhabiliter durablement les minorités. Pour ce faire, l'auteur attribue un rôle inédit aux marginalisés de l'Histoire dans le récit génésiaque.

\section{Conquérir une place dans le mythe}

L'entreprise de réécriture - ou de contre-écriture - amorcée par Raphaël Confiant du premier texte de création de l'humanité cristallise une telle efficace. Dans le premier chapitre de la Bible, l'ultime création de Dieu est l'Homme entendu comme modèle universel. Il faut en effet attendre la fin du vingt-septième verset pour observer une rupture, et la scission des êtres en deux sexes opposés :

Dieu dit : «Faisons l'homme à notre image, comme notre ressemblance, et qu'ils dominent sur les poissons de la mer, les oiseaux du ciel, les bestiaux, toutes les bêtes sauvages et toutes les bestioles qui rampent sur la terre. »

Dieu créa l'homme à son image,

à l'image de Dieu il le créa, homme et femme il les créa (Gn1, 26-27). 
Contrairement à la tradition yahviste, d'après laquelle la femme est tirée de l'homme et modelée à partir de sa côte (Gn 2, 22), la tradition élohiste présente donc "simultanément et explicitement la création de l'homme et de la femme» puisqu' " un même verset réunit l'annonce de la création et celle de la différence des sexes, complétant ainsi la première proposition anthropologique relative à l'image : l'homme mâle, d'une part, la femme, de l'autre, sont identiquement faits à l'image de Dieu et comme à sa ressemblance »(Pelletier, 1995, p. 35). Si, dans La Vierge du Grand Retour, nous retrouvons bien l'amorce injonctive « Faisons » (VGR, 15), la création qu'elle engage ne concerne ni l'Homme - au sens universel - ni l'homme au sens générique - mais la «négresse Philomène » (VGR, 15). Ainsi, après la nomination de la lumière en « devant-jour » $(V G R, 15)$ et des ténèbres en «brune du soir » $(V G R, 15)$ :

Dieu ajouta : "Faisons la négresse Philomène à notre image, comme notre ressemblance» (VGR, 15).

On notera ici l'introduction inattendue d'un article défini féminin. Contrairement à l'hypotexte biblique, une restriction générique est donc immédiatement opérée. Dès lors, la création, dans le roman, ne concerne pas l'humanité entière, mais une partie d'elle seulement, à savoir les femmes. On relèvera par ailleurs l'utilisation du terme fortement connoté de "négresse » qui, dans le contexte martiniquais, nous renvoie simultanément à une codification raciale et à une hiérarchisation sociale.

Plus tardive que celle de Philomène, l'apparition de l'« homme » sur la scène diégétique contrarie nos horizons de lecture, moins modulés par les versets de Gn1 que par des siècles de lectures abusives de Gn2 qui envisagent Ève comme une créature seconde et secondaire ${ }^{32}$ :

Dieu créa aussi Rigobert mais ne le bénit point. Dieu dit: 'Qu'étcétéra de millions d'années de maudition pèsent sur l'écale de ta race!' »(VGR, 16).

32 Le modèle constitué par Gn2 a conduit nombre de docteurs et de pères de l'Église à considérer la femme comme inférieure à l'homme. Deux textes majeurs de saint Paul argumenteront d'ailleurs en ce sens : la Première épître à Timothée, et la Première épître aux Corinthiens. D'après l'apôtre, «Ce n'est pas l'homme en effet qui a été tiré de la femme, mais la femme de l'homme ; et ce n'est pas l'homme, bien sûr, qui a été créé pour la femme, mais la femme pour l'homme » (1 Co 11-17). 
L'adverbe « aussi » exprime moins, dans le contexte du discours, une relation d'égalité avec la créature façonnée précédemment qu'il n'engage l'idée d'une ennuyeuse réitération du procès. L'arrivée de Rigobert ne consacre aucun couronnement. Acte de création et commandement divin sont comme expédiés. La prescription divine se voit réduite à une simple locution adverbiale (" étcétéra »). Toute solennité est bafouée. Yahvé-Dieu ne renouvelle d'ailleurs pas sa bénédiction, mais lance une malédiction ${ }^{33}$. Philomène seule est d'ailleurs investie par Yahvé-Dieu d'une autorité souveraine, et reçoit la maîtrise du petit monde en réduction figuré par l'ensemble des quartiers de Fort-de-France : "Que [la négresse Philomène] commande aux hommes de peine, aux marins en dérade, aux joueurs de serbi et de dominos, aux djobeurs du Grand Marché, aux dockers et à l'ensemble des femelles de céans!' »(VGR, 15). De sorte que dans la Genèse de Raphaël Confiant, la catégorie qui, à la suite d'une longue tradition de commentaires, est d'ordinaire appréhendée comme «dominante », fait l'objet d'un mouvement thématique dévalorisant. Si l'on peut légitimement parler, à propos de Philomène, d'une véritable «promotion » (GenetTe, 1982, p. 490), Rigobert, quant à lui, fait l'objet d'une remarquable « destitution ».

L'inflexion axiologique que l'écrivain fait subir à son texte est moins déterminée par le système de valeurs établi par son hypotexte premier Gn1, comme nous l'avons observé, ne marque aucun distinction initiale entre l'homme et la femme et, ce faisant, n'instaure entre eux aucun rapport de hiérarchie - que par le système symbolique de culture qui détermine et organise, de manière inégalitaire, les catégories du féminin et du masculin sur la base d'un autre hypotexte, ici souterrain : Gn2. Dans le récit de Confiant, la créature seconde, et secondaire, n'est plus la femme, mais l'homme. Ce faisant, les transformations sémantiques ne sauraient relever du seul régime ludique : en attribuant, selon les propres critères adoptés par un certain canon anthropologique, à «la négresse Philomène », paradigme de la minorité ${ }^{34}$ dans la mesure où elle est une femme noire de catégorie sociale basse, une dignité unique, l'opération relève simultanément du régime polémique. Une telle

33 On notera d'ailleurs la dimension particulièrement réductrice de la valeur de la suite l'énoncé. Tout n'est ici que négation : "Je ne t’offre ni herbes rares ni semences mirifiques qui sont sur la surface de la terre. » $(V G R, 16)$.

$34 \mathrm{La}$ 《 minorité » est ici entendue en tant que «catégorie ». Une telle catégorie englobe tous les individus assignés par le régime colonial au rang de "mineur », de « secondaire », de «moindre importance » dans la société, et relégué par lui dans les marges du discours officiel. Leur voix est longtemps restée condamnée au silence. 
opération vaut alors pour double pratique transvalorisationnelle ${ }^{35}$ aboutissant $\mathrm{au}$ 《 renversement complet d'un système de valeurs » (GENETTE, 1982, p. 483). La phraséologie génésiaque de Raphaël Confiant épouserait ainsi autant les formes d'un discours postcolonial que celles d'un discours féministe ${ }^{36}$. Tous deux ont d'ailleurs pour objet de réhabiliter la catégorie des marginalisés en face de celle des dominants. Selon l'appréciation de Spivak ${ }^{37}$, les femmes sont, dans de nombreuses sociétés, très souvent reléguées à une situation d'« Autre » marginalisé et métaphoriquement « colonisé » (SPIVAK G, 2006). Elles partagent avec lui « une expérience intime des politiques d'oppression et de répression» (AshCroft, Griffiths et Tiffin, 2012, p. 204). En nous offrant une lecture tout à la fois féministe (puisque se trouve valorisé le "féminin » au détriment du « masculin ») et postcoloniale (puisque le « premier homme » est une "négresse »), la Genèse de Confiant inverse les structures dominantes de nos sociétés ${ }^{38}$ - qui sont en grandes parties construites et véhiculées par l'image que l'on se fait traditionnellement de la Création - et formule un message politique fort.

La (les) «Genèse(s) » de Raphaël Confiant et de Patrick Chamoiseau se présentent comme un espace de rencontre entre le mythe et l'Histoire au sein duquel diverses opérations sont tour à tour ou simultanément effectuées :

35 Nous employons ce terme dans le sens où l'entend Genette à partir de la «transvaluation » nietzchéenne, c'est-à-dire comme une « opération d'ordre axiologique, portant explicitement ou implicitement attribuée à une action ou à un ensemble d'actions », GenetTe G., Palimpsestes, op.cit., p. 483.

36 Jacqueline Bardolph rappelle en effet que les problématiques qui traversent les théories féminines et les théories postcoloniales offrent à plusieurs égards le dessin de lignes parallèles. Voir Bardolph J., Études postcoloniales et littérature, op.cit., p. 45. Par ailleurs, les stratégies respectivement mises en place par l'une et l'autre théorie - telles que la réappropriation d'un discours dominant et son retournement - s'entrecroisent constamment. Voir Ashcroft B., Griffiths G. et Tiffin H., L'Empire vous répond : théorie et pratique des littératures post-coloniales, Mathieu-Joв M. et Serra J.-Y. (trad. fr.), Pessac, Presses universitaires de Bordeaux, coll. «Sémaphores », 2012, p. 203-206.

37 Métaphoriquement « colonisées », les femmes sont alors obligées de mener une véritable « guérilla » contre la domination impériale. Tout à la fois ancrées et exclues dans et de cet imperium, leur statut s'avère profondément ambivalent. Pour une analyse plus complète, nous renvoyons aux travaux de Spivak. Voir SpIVAK G. C., En d'autres mondes, en d'autres mots : essais de politique culturelle, Françoise Boullot (trad.fr.), Paris, Payot, 2009 [Spivak G. C., In otherworlds : essays in cultural politics, New York, Routledge, coll. "Routledge-classics ", 2006].

38 Les discours féministes et postcoloniaux ont en commun de chercher tous deux à " réhabiliter les marginalisés face aux dominants, et, dès leur émergence, ont tenté d'inverser les structures dominantes », Ashcroft B., Griffiths G. et Tiffin H., L’Empire vous répond, op.cit., p. 204-205. 
tentative d'élaboration d'un mythe originel manquant; reconfiguration des symboles pour dire - ou tenter de dire - « l'expérience de la diaspora noire, aspirée par une sorte de vide transatlantique "(Clavaron, 2012, p. 30); lutte amorcée contre une lecture institutionnelle de l'Histoire; mais aussi : contestation des structures dominantes d'une société donnée.

Contrairement à ce que racontent les Écritures, la Création du monde, dans Biblique des derniers gestes, ne remonte pas aux temps immémoriaux. D'emblée, elle est ancrée dans l'Histoire des Caraïbes dont l'origine, trop souvent « raturé[e]» (Glissant, 1998, p. 265) des mémoires et bannie des registres officiels, se confond avec l'« attentat colonial » (Chamoiseau, 1997, p. 200). La Genèse élaborée par Chamoiseau illustre le crime qui présida à la fondation de la communauté antillaise. Puisant aux pages les plus inattendues du Livre pour qui cherche à composer une nouvelle Genèse, l'écrivain martiniquais - en empruntant largement à l'imaginaire apocalyptique pour raconter le voyage transatlantique de son "vieux guerrier» au fond de la cale d'un bateau négrier -, traduirait ainsi l'urgence qu'il y aurait à trouver de nouveaux référents symboliques pour (re)fonder une " nouvelle entité collective » (Chamoiseau, 1997, p. 202). Le substrat biblique devient un précieux tremplin sur lequel s'appuyer pour inscrire, par le Verbe et dans la Lettre, l'Histoire dans le mythe et le mythe dans l'Histoire afin d'en « ouvrir » et d'en « achever» le sens (BDG, 773).

La Genèse apocalyptique de Patrick Chamoiseau conserve toute l'ambiguïté d'une véritable eschatologie. Rien ne l'empêche, à ce titre, d'ambitionner la même efficience pratique que n'importe quel récit cosmogonique - derrière l'anéantissement, se dissimule bien la présupposition d'une Création nouvelle... L'expérience historique est toute entière transformée en un "temps d'illumination». Ce dernier, nécessairement conjoncturel et transitoire, embrasse autant qu'il reflète les dynamiques respiratoires du monde encore balbutiant. La naissance (ou la renaissance) de Balthazar Bodule-Jules coïncide avec ce moment de « pile-exacte » $(B D G, 62)$ où l'univers entier est éprouvé comme une réalité composée en perpétuelle recomposition. Cette Apocalypse génésiaque, qui raconte moins l'origine du monde que celle du "Chaos-monde ", aura permis une transmutation des valeurs négatives de l'expérience traumatique initiale.

Toutefois, loin de « déborde[r] les limites de l'expérience pour rejoindre ce qui, précédant celle-ci, la fonde et lui confère un sens » (Pelletier, 1995, p. 28), les Genèses antillaises, prisonnières des tragédies de l'Histoire, 
déploient obliquement un certain nombre d'arguments critiques. Une tonalité polémique - non dépourvue d'humour - imprègne tout spécifiquement les lignes de la Genèse parodique de Raphaël Confiant qui n'inscrit pas sa Création dans un ailleurs inaccessible à la connaissance humaine, mais dans un lieu géographique bien défini, et circonscrit - l'île de la Martinique. De nouvelles frontières de la Création hébraïque sont ainsi esquissées, dont la "négresse Philomène », emblème du "minoritaire ", Noire et Femme, en est la souveraine créature. Le détournement de la lettre biblique et de son esprit œuvre activement au renversement des préjugés établis par des millénaires de lectures abusives. Revêtant l'apparence du "contre-discours » tel que l'a théorisé Helen Tiffin (1987, p. 17-34), la Genèse subversive de Raphaël Confiant répond à l'enjeu politique de "donne[r] une voix à qui n'en a pas » (Calvino, 1984, p. 82) dans l'Histoire et dans le mythe.

\section{Bibliographie :}

La Bible de Jérusalem (1998), L’École biblique de Jérusalem (trad. fr.) Paris, Pocket, coll. « Pocket », n¹0456, Nouv. éd. rev. et cor., 2013.

ASHCROFT B., GRIFFITHSG. ET TIFFIN H., L'Empire vous répond. Théorie et pratique des littératures post-coloniales, Martine Mathieu-Job et Jean-Yves Serra (trad. fr.), Pessac, Presses universitaires de Bordeaux, coll. «Sémaphores », 2012.

AUZAS N., "L'imaginaire, un chant de bataille antillais ", Iris, n³2, coll. "Espaces mythiques », 2011.

BARDOLPH J., Études postcoloniales et littérature, Paris, Honoré Champion, coll. «Unichamp-essentiel », n¹0, 2002.

CALVINO I., La Machine littérature. Essais, ORCEL M. ETWAHF. (trad. fr.), Paris, Éditions du Seuil, coll. " Pierres vives », n¹20, 1984.

CHAMOISEAU P., Écrire en pays dominé, Paris, Gallimard, 1997.

CHAMOISEAU P., Biblique des derniers gestes, Paris, Gallimard, 2002.

CHAUVIN D., "Apocalypse », Dictionnaire des mythes littéraires, in Dictionnaire des mythes littéraires (1988), Pierre Brunel (dir.), Paris Monaco, Éditions du Rocher, Nouv. éd. augm., 1994.

CHAUVIN D., "Mémoire et mythe », Questions de mythocritique. Dictionnaire, CHAUVIN D., SIGANOS A. et WALTER PH. (dir.), Éditions Imago, 2005.

CHAUVIN D., « Biblique des derniers gestes : les enjeux d'un titre », Autour de Patrick Chamoiseau, SWOBODA T., WIERZBOWSKA E. et WRONSKA O. (dir.), Fundacja Rozwoju Uniwersytetu Gdanskiego, Cahiers ERTA, t. 1, 2008.

CLAVARON Y., "Autorité des empires de l'Atlantique », Les Empires de l'Atlantique XIX ${ }^{\text {- }}$ $\mathrm{XXI}^{\mathrm{e}}$ siècles. Figures de l'autorité impériale dans les lettres d'expression européenne de l'espace atlantique, Clavaron Y. et Moura J.-M. (dir.), Bécherel Les Perséides, 2012. 
CONFIANT R., cité par Raymond RELOUZAT DANS RELOUZAT R., Tradition orale et imaginaire créole, Martinique, Ibis Rouge, Presses universitaires créoles-GEREC, 1998.

CONFIANT R., «La créolité contre l'enfermement identitaire », Multitudes, $n^{\circ} 22$, mars 2005, p. 179-185.

CONFIANT R., La Vierge du Grand Retour (1996), Paris, Gallimard, coll. "Folio », n $4602,2007$.

FRATTA C. et GIAUFRET A., « La Vierge du Grand Retour de Raphaël Confiant et A barca di a Madonna de Ghjacumu Thiers ou de la centralité convergente des marge ", Publifarum, Dipartimento di Lingue e Culture Moderne, Univerità di Genova, 2009.

GANNIER O., «Antilles francophones », La Bible dans les littératures du monde, PARIZET S. (dir.), Paris, Cerf, vol.1, 2016.

GENeTTE G., Palimpsestes, La littérature au second degré, Paris, Seuil, coll. «Points essais ", n²57, 1982.

GIRAUD J.-P., «Typologie des mythes », Questions de mythocritique. Dictionnaire, CHAUVIN D., SIGANOS A. et WALTER PH. (dir.), Éditions Imago, 2005.

GLISSANT E., Poétique de la Relation, Paris, Gallimard, 1990.

GLISSANT E., Faulkner, Mississipi (1996), Paris, Gallimard, coll. « Folio/Essais », 1998.

KRISTEVA J., Séméiotikè. Recherches pour une sémanalyse (1969), Paris, Éditions du Seuil, coll. «Points », n96, 1978.

PELLETIER A.-M., Lectures bibliques. Aux sources de la culture occidentale, Paris, Nathan Cerf, coll. « Réf. », 1995.

SPIVAK G. C., En d'autres mondes, en d'autres mots. Essais de politique culturelle, FRANÇOISE BOUILLOT (trad. fr.), Paris, Payot, 2009 [SPIVAK G. C., In otherworlds. Essays in cultural politics, New York, Routledge, coll. «Routledge-classics », 2006].

TIFFIN H., "Post-colonial literatures and counter-discourse », Kunapipi, Anna RUTHERFORD, Aarhus, Dangaroo Pres, nº 1987.

Submetido em: 24-10-2019

Aceito em: 21-11-2019 